\title{
Effect of alcohol and aldehyde dehydrogenase gene polymorphisms on alcohol-associated hypertension: the Guangzhou Biobank Cohort Study
}

\author{
Wei Sen Zhang ${ }^{1,4}$, Lin $\mathrm{Xu}^{2,4}$, Catherine Mary Schooling ${ }^{2}$, Chao Qiang Jiang ${ }^{1}$, Kar Keung Cheng ${ }^{3}$, Bin Liu ${ }^{1}$ \\ and Tai Hing Lam $^{2}$
}

The effects of alcohol dehydrogenase (ADH) 2 and aldehyde dehydrogenase (ALDH) 2 genotypes on the alcohol-blood pressure association are unclear. We examined the association of ADH2 or ALDH2 genotypes with blood pressure in older Chinese men. Based on the Guangzhou Biobank Cohort Study (GBCS), 4792 men with valid ADH2, ALDH2 genotypes were included, and genotyping of rs1229984 ADH2 and rs671 ALDH2 (AA, AG/GA or GG) was performed using a Sequenom Mass-Array platform. Information on socio-demographics and lifestyle factors, including alcohol use, was obtained from a questionnaire, and blood pressure was measured. Among alcohol drinkers, systolic and diastolic blood pressure (SBP and DBP) and mean arterial pressure (MAP) were highest for men with the GG ADH2 genotype (136.6, 77.9 and $97.5 \mathrm{~mm} \mathrm{Hg}$, respectively), followed by those with the (AA/AG ADH2 + GG ALDH2) genotype $(133.4,77.6$ and $96.2 \mathrm{~mm} \mathrm{Hg}$, respectively) and then the (AA/AG ADH2 + AA/AG ALDH2) genotype $(\mathrm{SBP}=132.6, \mathrm{DBP}=76.6$ and $\mathrm{MAP}=95.2 \mathrm{~mm} \mathrm{Hg})(P$ for trend ranged $0.025-0.035)$. After adjustment for potential confounders, as well as frequency or amount of alcohol use, men with the GG ADH2 genotype were more likely to have hypertension (odds ratio $(O R)=1.62,95 \%$ confidence interval $1.15-2.28$ ) as were men with the (AA/AG ADH2 + AA/AG ALDH2) genotype $(\mathrm{OR}=1.40,95 \%$ confidence interval $1.01-1.96)$ compared with men with the (AA/AG ADH2 + GG ALDH2) genotype). ADH2 or ALDH2 genotypes were unrelated to hypertension among those who never drink alcohol. ADH2 genotype influences blood pressure and risk of hypertension among male alcohol drinkers, suggesting that the hypertensive effect of alcohol is due to ethanol rather than acetaldehyde.

Hypertension Research (2013) 36, 741-746; doi:10.1038/hr.2013.23; published online 25 April 2013

Keywords: alcohol; aldehyde dehydrogenase gene polymorphisms; blood pressure

\section{INTRODUCTION}

Alcohol consumption is increasingly popular in China. ${ }^{1}$ Based on a WHO report, alcohol consumption per capita in pure ethanol for Chinese adults was 1.03 litres in 1970, and rose to 4.5-5.2 litres in 1996-2001..$^{2-4}$ The increasing use of alcohol has caused a great health, financial and social burden in China, yet alcohol consumption is continuing to rise. Local research is urgently needed to provide supporting evidence for developing a policy for alcohol restriction to improve public health.

Alcohol has been linked to hypertension in previous studies, and the association was partly explained by genetic variation in alcohol metabolism. ${ }^{5}$ Studies in Japanese and other East Asian populations have compared the alcohol-blood pressure relationships among individuals with varying activities of alcohol-metabolizing enzymes, such as alcohol dehydrogenase $(\mathrm{ADH})$ and aldehyde dehydrogenase $(\mathrm{ALDH}),{ }^{6,7}$ and have shown important roles for these enzymes in the metabolism of alcohol in the body. ${ }^{8}$ After alcohol intake, ethanol is firstly metabolized to acetaldehyde then to acetic acid through several mechanisms. The main enzymes involved in the metabolic process are ADH2 and ALDH2 enzymes. ${ }^{9-11}$ The activities of these enzymes differ with genetic polymorphisms, leading to different effects of alcohol consumption on health. The GG and AA ALDH2 genotypes encode the active and the inactive subunit, respectively. ${ }^{12,13}$ The AA ALDH2 genotype results in higher blood acetaldehyde levels after alcohol intake, leading to facial flushing, palpitations and nausea. ${ }^{7,14}$ A metaanalysis of studies using ALDH2 single-nucleotide polymorphisms as an instrumental variable for alcohol use showed that alcohol significantly increased blood pressure and risk of hypertension in individuals of AA genotype. ${ }^{5}$ However, this meta-analysis was designed to assess the effect of alcohol use, and specifically ethanol, on blood pressure, but the effect of the ADH2 genotype on blood pressure was not assessed. As the isozymes of $\mathrm{ADH} 2$ encoded by the

${ }^{1}$ Molecular Epidemiological Research Centre, Guangzhou No. 12 Hospital, Guangzhou, China; ${ }^{2}$ Department of Community Medicine, School of Public Health, University of Hong Kong, Hong Kong and ${ }^{3}$ Public Health, Epidemiology, and Biostatistics, University of Birmingham, Birmingham, UK

Correspondence: Professor CQ Jiang, Molecular Epidemiological Research Centre, Guangzhou No. 12 Hospital, Guangzhou, China.

E-mail: cqjiang@hkucc.hku.hk

${ }^{4}$ Joint first author.

Received 5 October 2012; revised 13 December 2012; accepted 10 January 2013; published online 25 April 2013 
AA and AG genotypes have about 200 and 100 times higher ethanol oxidizing capacity, respectively, than the GG ADH2 genotype, ${ }^{15}$ the effect of alcohol on blood pressure should vary with $\mathrm{ADH} 2$ genotype. A previous study on men found that subjects with the GG genotype of $\mathrm{ADH} 2$ had higher blood pressure than those with the GA or AA genotype, ${ }^{16}$ but this study did not consider whether the association varied with ALDH2 genotype, despite the effects of ALDH2 on alcohol metabolism. Figure 1 shows the likely exposures for different combinations of $\mathrm{ADH} 2$ and $\mathrm{ALDH} 2$ genotypes. Individuals with an inactive $\mathrm{ADH} 2$ (GG) genotype metabolize alcohol slowly and have high ethanol exposure on alcohol use. Individuals with an inactive ALDH2 (AA or AG) genotype metabolize acetaldehyde slowly and have high acetaldehyde exposure on alcohol use. Individuals with an active $\mathrm{ADH} 2$ (AA or $\mathrm{AG}$ ) genotype and an active ALDH2 (GG) genotype metabolize both ethanol and acetaldehyde quickly and have high acetic acid exposure on alcohol use. We hypothesized that in alcohol drinkers, those with an inactive ADH2 (GG) genotype have higher levels of ethanol than those with an active genotype and that this genotype may be associated with a higher risk of hypertension after alcohol drinking.

In the GBCS, we examined the associations of ADH2 and ALDH2 genotypes with blood pressure in men, and whether the associations varied with drinking status (alcohol drinker or never drinker).

\section{METHODS}

\section{Ethics statement}

All subjects had given informed consent before participating. The study has ethics approval from the Guangzhou Medical Ethics Committee of the Chinese Medical Association, Guangzhou, China.

\section{The details of the GBCS}

The GBCS is a 3-way collaboration among the Guangzhou No. 12 Hospital, the Universities of Hong Kong and Birmingham. The details have been reported elsewhere. ${ }^{17}$ Briefly, participants were recruited from the 'Guangzhou Health and Happiness Association for the Respectable Elders', a community social and welfare association unofficially aligned with the municipal government. This is a large unofficial organization with more than 150 branches throughout Guangzhou. The membership is open to Guangzhou residents aged 50 years or above for a nominal fee of 4 CNY ( 50 US cents) per month. Members of the Guangzhou Health and Happiness Association for the Respectable Elders account for about 7\% of older permanent residents in Guangzhou. Approximately one-third of them participated in the GBCS if they were capable of consenting, ambulatory and not receiving treatment modalities that, if omitted, may result in immediate life-threatening risk, such as chemotherapy or radiotherapy for cancer, or dialysis for renal failure. As participants in the GBCS could receive a free medical examination, the response rate was very high (about $95 \%$ ). About $5 \%$ of the eligible subjects refused to participate, particularly men, because of a cultural unwillingness to give blood due to an associated loss of 'shung qi' or 'life energy', or because of job commitments. The baseline examinations were conducted in three phases:

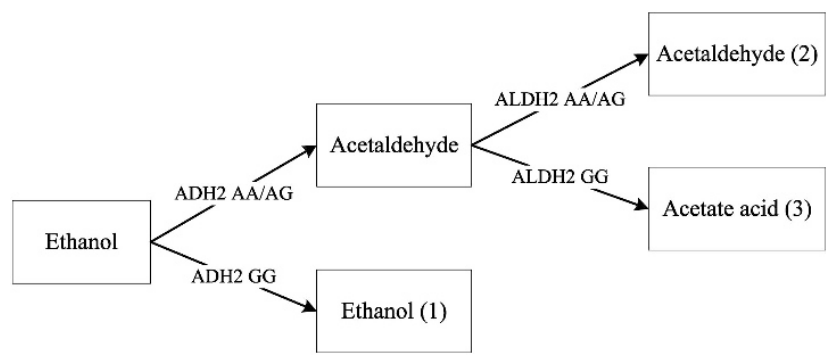

Figure 1 Effect of $A D H 2$ and ALDH2 genotype on alcohol metabolism in human. phase 1 from September 2003 to November 2004, phase 2 from April 2005 to May 2006 and phase 3 from September 2006 to January 2008. The second examination started in 2008 and in progress.

Computer based face-to-face interview and detailed physical examinations were done at baseline recruitment. Information on alcohol consumption was obtained in terms of frequency and usual amount per occasion for specific types of alcoholic beverage. Seated blood pressure was measured three times using an automated sphygmomanometer (Omron 705CP; Omron Corp., Kyoto, Japan), which had been previously validated by comparison with the manual mercury sphygmomanometer, ${ }^{18}$ and the last two measurements were averaged for analysis.

Blood samples were collected following $8-10 \mathrm{~h}$ overnight fasting. Blood samples for DNA extraction were available in phase 3 at baseline and in phases 1 and 2 at follow-up. DNA exaction was performed at the laboratory center of the GBCS in Guangzhou 12th Hospital. ${ }^{19}$ DNA was either extracted at baseline from fresh blood using a standard phenol-chloroform extraction procedure or was extracted from blood or buffy coat previously stored at $-80^{\circ} \mathrm{C}$ using a standard magnetic bead extraction procedure. single-nucleotide polymorphism analysis was performed using a Sequenom Mass-Array platform, which amplifies DNA with specific primers, deactivates remaining nucleotide triphosphates by phosphatase treatment, performs a single base primer extension step and identifies allele-specific extension products using mass spectroscopy.

\section{Outcomes}

After resting for $5 \mathrm{~min}$, seated SBP and DBP was measured three times using an automated sphygmomanometer (Omron $705 \mathrm{CP}$ ), which had been previously validated by comparison with the manual mercury sphygmomanometer, ${ }^{18}$ and at least $1 \mathrm{~min}$ rest interval was required between each measurement. The last two measurements were averaged for data analysis in the present study. Outcomes considered were SBP, DBP, pulse pressure, defined as $\mathrm{SBP}-\mathrm{DBP}$, MAP defined as $1 / 3 \times(\mathrm{SBP}+\mathrm{DBP} \times 2)$ and hypertension defined as use of antihypertensive medication or SBP/DBP $\geqslant 140 / 90 \mathrm{~mm} \mathrm{Hg}$.

\section{Exposures}

Exposures considered were rs671 ALDH2 genotype, rs1229984 ADH2 genotype and their combination, as set out in Figure 1, which shows how ADH2 and ALDH2 were combined to represent ethanol, acetaldehyde or acetic acid exposure.

\section{Statistical analysis}

We used genetic polymorphism of alcohol metabolism enzyme (ALDH2 and $\mathrm{ADH} 2$ genotypes) as the instrumental variable to obtain an unbiased estimation of the effect of alcohol on blood pressure. As genetic differences are determined before birth, these are unlikely to be associated with exogenous exposures or environmental factors. Thus the estimation might not be confounded by common hypertensive risk factors, such as smoking or sodium intake, as in other observational studies. Multivariable linear regression was used to assess differences in continuous variables between genotypes with adjustment for potential confounders, giving adjusted means and 95\% confidence intervals, as well as $P$-value for trend. Logistic regression was used to assess the association of $\mathrm{ADH} 2$ and $\mathrm{ALDH} 2$ polymorphism with hypertension with adjustment for potential confounders. We assessed whether the association of ADH2 with the outcomes varied with ALDH2 from the $P$-value for interaction. We similarly assessed whether the association of $\mathrm{ADH} 2$, ALDH2 or the combined ADH2/ALDH2 genotype with the outcomes varied with drinking status. Potential confounders were age, occupation (manual, nonmanual, others), education, International Physical Activity Questionnaire physical activity (active, moderate, and inactive), ${ }^{20}$ smoking (never, former and current), body mass index, fasting plasma glucose and total and high-density lipoprotein cholesterol. We excluded former drinkers when assessing the association of $\mathrm{ADH} 2$ and $\mathrm{ALDH} 2$ genotypes with blood pressure to reduce the 'abstainer error', as former drinkers might have quitted drinking because of ill-health including hypertension, and received antihypertensive medication. ${ }^{21}$ Statistical significance was defined as a two-sided $P$-value $<0.05$. Data analysis was performed using STATA 10.1 (Stata Corp LP, College Station, TX, USA). 


\section{RESULTS}

There were 4792 men with valid $\mathrm{ADH} 2$ and ALDH2 genotypes and complete information about all potential confounders. The AA, AG and GG genotype frequencies of ADH2 and ALDH2 were 52.7\%, $40.2 \%$ and $7.1 \%$, and $8.6 \%, 41.6 \%$ and $49.8 \%$, respectively. The Hardy-Weinberg equilibrium test showed no variance between the population under study and the general population $(P=0.77$ for $\mathrm{ADH} 2$ and 0.99 for ALDH2 genotypes).

Table 1 shows that the $\mathrm{ADH} 2$ genotype was unrelated to drinking status or frequency, or to other risk factors, such as smoking, physical activity, education, occupation, age, body mass index, total and highdensity lipoprotein-cholesterol, triglycerides and fasting glucose. Current drinking and higher alcohol use $(\geqslant 140 \mathrm{~g}$ per week) were more common among men with the GG ADH2 genotype $(P=0.050$ and 0.026 , respectively) than men with the other $\mathrm{ADH} 2$ genotypes. Current drinking, frequent alcohol drinking $(\geqslant 5$ per week) and higher alcohol use ( $\geqslant 140 \mathrm{~g}$ per week) were all more common among men with the GG than the AA or AG ALDH2 genotypes (all $P<0.001)$. Men with the AA ALDH2 genotype were more likely to have had manual occupations $(P=0.040)$ and lower total and high-density lipoprotein-cholesterol $(P=0.036$ and 0.005, respectively).

There was no evidence that the association of $\mathrm{ADH} 2$ with the outcomes varied with ALDH2, probably because of insufficient sample size ( $P$-values ranged from 0.19 to 0.44 ; table not shown). Similarly, there was no evidence that the association of $\mathrm{ADH} 2$ or ALDH2 with the outcomes varied with drinking status; however, the association of the combined ADH2/ALDH2 group with some outcomes did vary with drinking status (SBP $P=0.035$, DBP $P=0.035$ and MAP $P=0.025$ ). Table 2 shows that in never drinkers, $\mathrm{ADH} 2$ and ALDH2 were unrelated to SBP, DBP, pulse pressure or MAP. Among current drinkers, compared with the AA or AG ADH2 genotype, men with the GG genotype had higher SBP and pulse pressure $(P<0.05)$. ALDH2 genotypes were unrelated to SBP, DBP, pulse pressure or MAP. After adjusting for multiple potential confounders and frequency of drinking, SBP, DBP and MAP were highest for men with the GG ADH2 genotype, followed by those with the (AA/AG $\mathrm{ADH} 2+\mathrm{GG}$ ALDH2) genotype and then the (AA/AG ADH2 + AA/ AG ALDH2) genotype ( $P$ for trend ranged 0.025-0.035) (Table 2). Sensitivity analysis with additional adjustment for amount of alcohol

Table 1 Characteristics by ADH2 and ALDH2 genotypes in older Chinese men

\begin{tabular}{|c|c|c|c|c|c|c|c|c|c|}
\hline & & \multicolumn{4}{|c|}{$A D H 2(r s 1229984, \mathrm{n}=4792)$} & \multicolumn{4}{|c|}{$A L D H 2(r s 671, \mathrm{n}=4782)$} \\
\hline & & $A A$ & $A G$ & $G G$ & $P$ & $A A$ & $A G$ & $G G$ & $\mathrm{P}$ \\
\hline Cases, \% & & $2524(52.7)$ & $1927(40.2)$ & $341(7.1)$ & $0.77^{a}$ & $409(8.6)$ & $1990(41.6)$ & $2383(49.8)$ & $0.99^{a}$ \\
\hline \multirow[t]{3}{*}{ Drinking, $n(\%)$} & Never & $1350(53.5)$ & 955 (49.6) & $162(47.5)$ & 0.050 & 315 (77.0) & 1173 (58.9) & 969 (40.7) & $<0.001$ \\
\hline & Former & $139(5.5)$ & $122(6.3)$ & $20(5.9)$ & & $9(2.2)$ & $100(5.0)$ & $172(7.2)$ & \\
\hline & Current & 1035 (41.0) & $850(44.1)$ & 159 (46.6) & & $85(20.8)$ & 717 (36.0) & $1242(52.1)$ & \\
\hline \multirow[t]{6}{*}{ Frequency of drinking, $n(\%)$} & Never & $1350(53.5)$ & 955 (49.6) & $162(47.5)$ & 0.26 & 315 (77.0) & 1173 (58.9) & 969 (40.7) & $<0.001$ \\
\hline & $<1$ per month & $537(21.3)$ & $415(21.5)$ & $82(24.0)$ & & $70(17.1)$ & 432 (21.7) & $536(22.5)$ & \\
\hline & $<1$ per week & 99 (3.9) & $86(4.5)$ & $15(4.4)$ & & $5(1.2)$ & $83(4.2)$ & $110(4.6)$ & \\
\hline & 1-4 per week & $142(5.6)$ & $120(6.2)$ & $18(5.3)$ & & $4(1.0)$ & 78 (3.9) & $196(8.2)$ & \\
\hline & $\geqslant 5$ per week & $257(10.2)$ & 229 (11.9) & 44 (12.9) & & $6(1.5)$ & $124(6.2)$ & $400(16.8)$ & \\
\hline & Former drinkers & $139(5.5)$ & $122(6.3)$ & $20(5.9)$ & & $9(2.2)$ & $100(5.0)$ & $172(7.2)$ & \\
\hline \multirow[t]{3}{*}{ Amount of alcohol use, $n(\%)$} & Never & $1350(53.5)$ & 955 (49.6) & $162(47.5)$ & 0.026 & $315(77.0)$ & 1173 (58.9) & 969 (40.7) & $<0.001$ \\
\hline & $<140$ g per week & 1005 (39.8) & $852(44.2)$ & $154(45.2)$ & & $91(22.2)$ & 767 (38.5) & $1151(48.3)$ & \\
\hline & $\geqslant 140$ g per week & $169(6.7)$ & $120(6.2)$ & $25(7.3)$ & & $3(0.7)$ & $50(2.5)$ & $263(11.0)$ & \\
\hline \multirow[t]{2}{*}{ Smoking, $n(\%)$} & Never & $1010(40.0)$ & 769 (39.9) & $157(46.0)$ & 0.09 & $170(41.6)$ & $812(40.8)$ & 951 (39.9) & 0.74 \\
\hline & Ever & $1514(60.0)$ & $1158(60.1)$ & $184(54.0)$ & & $239(58.4)$ & $1178(59.2)$ & $1432(60.1)$ & \\
\hline \multirow[t]{3}{*}{ Physical activity, $n(\%)$} & Inactive & $216(8.6)$ & $126(6.5)$ & $33(9.7)$ & 0.09 & $39(9.5)$ & $160(8.0)$ & $178(7.5)$ & 0.13 \\
\hline & Moderate & $1006(39.9)$ & $785(40.7)$ & $131(38.4)$ & & $152(37.2)$ & $767(38.5)$ & $994(41.7)$ & \\
\hline & Active & $1302(51.6)$ & $1016(52.7)$ & $177(51.9)$ & & $218(53.3)$ & $1063(53.4)$ & $1211(50.8)$ & \\
\hline \multirow[t]{4}{*}{ Education, $n(\%)$} & Primary or below & $724(28.7)$ & $546(28.3)$ & $102(29.9)$ & 1.00 & $109(26.7)$ & $589(29.6)$ & $674(28.3)$ & 0.29 \\
\hline & Junior middle & $769(30.5)$ & $587(30.5)$ & $106(31.1)$ & & $121(29.6)$ & $600(30.2)$ & $734(30.8)$ & \\
\hline & Senior middle & $621(24.6)$ & $482(25.0)$ & $81(23.8)$ & & $112(27.4)$ & $505(25.4)$ & $567(23.8)$ & \\
\hline & College & $410(16.2)$ & $312(16.2)$ & $52(15.2)$ & & $67(16.4)$ & 296(14.9) & $408(17.1)$ & \\
\hline \multirow[t]{3}{*}{ Occupation, $n(\%)$} & Manual & $1285(50.9)$ & $931(48.3)$ & $188(55.1)$ & 0.12 & $217(53.1)$ & $1028(51.7)$ & $1153(48.4)$ & 0.040 \\
\hline & Nonmanual & $973(38.5)$ & 768(39.9) & $120(35.2)$ & & $156(38.1)$ & 731(36.7) & $970(40.7)$ & \\
\hline & Others & $266(10.5)$ & 228 (11.8) & $33(9.7)$ & & $36(8.8)$ & $231(11.6)$ & 260 (10.9) & \\
\hline Age, year (s.d.) & & $63.6(6.9)$ & $63.5(6.9)$ & $63.6(6.9)$ & 0.95 & $63.4(6.9)$ & $63.6(7.0)$ & $63.5(6.9)$ & 0.66 \\
\hline Body mass index, $\mathrm{kg} \mathrm{m}^{-2}$ (s.d.) & $23.5(3.2)$ & $23.5(3.1)$ & $23.7(3.0)$ & 0.56 & $23.5(3.0)$ & $23.5(3.1)$ & $23.5(3.2)$ & 0.95 & \\
\hline Total cholesterol, mmoll-1 (s.d.) & $5.59(1.06)$ & $5.57(1.08)$ & $5.52(0.92)$ & 0.49 & $5.45(0.99)$ & $5.57(1.07)$ & $5.60(1.07)$ & 0.036 & \\
\hline Triglycerides, mmoll-1 (s.d.) ${ }^{\mathrm{b}}$ & $1.43(1.73)$ & $1.40(1.73)$ & $1.40(1.70)$ & 0.35 & $1.42(1.67)$ & $1.40(1.71)$ & $1.43(1.75)$ & 0.35 & \\
\hline HDL cholesterol, mmoll-1 (s.d.) & $1.52(0.37)$ & $1.51(0.37)$ & $1.50(0.35)$ & 0.82 & $1.47(0.35)$ & $1.50(0.37)$ & $1.53(0.38)$ & 0.005 & \\
\hline Fasting glucose, mmoll ${ }^{-1}(\text { s.d. })^{b}$ & $5.59(1.21)$ & $5.59(1.22)$ & $5.57(1.20)$ & 0.92 & $5.57(1.21)$ & $5.58(1.21)$ & $5.60(1.22)$ & 0.68 & \\
\hline
\end{tabular}

Abbreviations: $A D H$, alcohol dehydrogenase; ALDH, aldehyde dehydrogenase.

aHardy-Weinberg equilibrium test.

bData was expressed as geometric means (s.d.). 
Table 2 Association of ADH2 and ALDH2 genotypes with blood pressure in older Chinese men

\begin{tabular}{|c|c|c|c|c|c|c|c|}
\hline & & & \multirow[b]{2}{*}{$n$} & \multicolumn{4}{|c|}{ Adjusted means $(95 \% \mathrm{Cl})$} \\
\hline & & & & Systolic BP & Diastolic BP & $P P$ & MAP \\
\hline \multicolumn{8}{|c|}{ Never drinkers } \\
\hline \multicolumn{3}{|c|}{ ADH2(rs1229984) } & 2467 & & & & \\
\hline Group 1 & \multicolumn{2}{|l|}{$\mathrm{AA}$} & 1350 & $132.6(131.3,133.9)$ & $75.2(74.5,75.9)$ & $57.4(56.4,58.3)$ & $94.3(93.5,95.2)$ \\
\hline Group 2 & \multicolumn{2}{|l|}{$A G$} & 955 & $133.0(131.5,134.5)$ & $75.0(74.2,75.8)$ & $57.9(56.9,59.0)$ & $94.3(93.4,95.3)$ \\
\hline \multirow[t]{2}{*}{ Group 3} & \multicolumn{2}{|l|}{ GG } & 162 & $131.4(128.3,134.5)$ & $73.3(71.7,75.0)$ & $58.0(55.9,60.2)$ & $92.7(90.7,94.7)$ \\
\hline & \multicolumn{3}{|c|}{$P$ for trend } & 0.79 & 0.09 & 0.36 & 0.28 \\
\hline \multicolumn{3}{|c|}{ ALDH2 (rs671) } & 2457 & & & & \\
\hline Group 1 & \multicolumn{2}{|l|}{$\mathrm{AA}$} & 315 & $132.2(129.9,134.5)$ & $74.5(73.3,75.8)$ & $57.7(56.0,59.3)$ & $93.7(92.3,95.2)$ \\
\hline Group 2 & \multicolumn{2}{|l|}{$A G$} & 1173 & $132.9(131.5,134.3)$ & $75.1(74.3,75.8)$ & $57.8(56.9,58.8)$ & $94.3(93.4,95.2)$ \\
\hline \multirow[t]{2}{*}{ Group 3} & \multicolumn{2}{|l|}{$\mathrm{GG}$} & 969 & $132.7(131.2,134.1)$ & $75.1(74.3,75.9)$ & $57.5(56.5,58.6)$ & $94.3(93.4,95.3)$ \\
\hline & \multicolumn{3}{|c|}{$P$ for trend } & 0.91 & 0.44 & 0.66 & 0.62 \\
\hline Interaction & $\mathrm{ADH} 2$ & ALDH2 & 2450 & & & & \\
\hline Group 1 & $A A+A G$ & $A A+A G$ & 1397 & $132.8(131.5,134.1)$ & $75.1(74.4,75.8)$ & $57.7(56.8,58.6)$ & $94.4(93.5,95.2)$ \\
\hline Group 2 & $A A+A G$ & GG & 891 & $132.7(131.2,134.2)$ & $75.2(74.4,76.0)$ & $57.5(56.4,58.5)$ & $94.4(93.4,95.4)$ \\
\hline \multirow[t]{2}{*}{ Group 3} & GG & Any genotype & 162 & $131.4(128.3,134.5)$ & $73.3(71.7,75.0)$ & $58.0(55.9,60.2)$ & $92.7(90.7,94.7)$ \\
\hline & \multicolumn{3}{|c|}{$P$ for trend } & 0.42 & 0.21 & 0.85 & 0.27 \\
\hline
\end{tabular}

Current drinkers

ADH2 (rs1229984)

Group $1 \quad$ AA

Group $2 \quad A G$

Group $3 \quad$ GG

$P$ for trend

ALDH2 (rs671)

Group $1 \quad$ AA

Group $2 \quad$ AG

Group 3 GG

Interaction ADH2

Group $1 \quad A A+A G$

Group $2 \quad A A+A G$

Group 3

$G G$

$P$ for trend

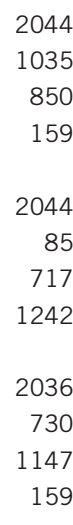

$130.7(126.0,135.3)$

$133.2(131.1,135.3)$

$133.5(131.7,135.3)$ 0.35

$132.6(130.5,134.7)$

$133.4(131.6,135.3)$

$136.6(133.3,140.0)^{*}$ 0.035

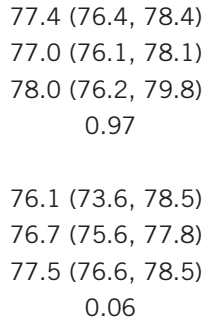

$77.4(76.4,78.4)$ $77.0(76.1,78.1)$

$78.0(76.2,79.8)$

0.97

$76.1(73.6,78.5)$

$76.7(75.6,77.8)$

$77.5(76.6,78.5)$ 0.06

$55.8(54.4,57.1)$

$56.1(54.8,57.5)$

$58.7(56.4,61.0)^{*}, \#$

0.039

$76.6(75.4,77.7)$

$77.6(76.6,78.6)^{*}$

$77.9(76.1,79.7)$

0.035
$54.6(51.4,57.8)$

$56.6(55.1,58.0)$

$56.0(54.7,57.2)$

0.91

$56.0(54.6,57.5)$

$55.8(54.6,57.1)$

$58.7(56.4,61.0)^{*}$, \#

0.15
$96.0(94.8,97.2)$

$95.8(94.5,97.0)$

$97.6(95.4,99.7)$

0.44

$94.3(91.3,97.3)$

95.5 (94.2, 96.9)

$96.2(95.0,97.4)$

0.13

$95.2(93.9,96.6)$

$96.2(95.0,97.4)$

$97.5(95.3,99.7)^{*}$ 0.025

Abbreviations: ADH, alcohol dehydrogenase; ALDH, aldehyde dehydrogenase, BP, blood pressure; Cl, confidence interval; HDL, high-density lipoprotein; PP, pulse pressure; MAP, mean arterial blood pressure.

Compared with group 1: ${ }^{*} P<0.05$. Compared with group 2: \# $P<0.05$.

adjustment for age, education, occupation, smoking, physical activity, body mass index, as well as fasting total cholesterol, triglyceride, HDL cholesterol, glucose and frequency of drinking (for current drinkers only).

use instead of frequency of drinking showed similar results (Table not shown).

Table 3 shows that among ever drinkers, men with the GG ADH2 genotype had higher odds of hypertension than those with the (AA/ $\mathrm{AG} \mathrm{ADH} 2+\mathrm{AA} / \mathrm{AG} \mathrm{ALDH} 2)$ genotype after adjusting for frequency of alcohol drinking and other risk factors $(\mathrm{OR}=1.62,95 \%$ confidence interval 1.15-2.28). Sensitivity analysis found similar results after adjusting for amount of alcohol drinking and other risk factors $(\mathrm{OR}=1.61$ (1.14-2.27)). Compared with (AA/AG ADH2 + GG $\mathrm{ALDH} 2$ ), the adjusted $\mathrm{OR}$ for GG ADH2 was 1.40 (1.01, 1.96). Among men with the AA/AG ADH2 genotype, the ALDH2 genotype was not significantly associated with hypertension. (Table not shown) Among never drinkers, $\mathrm{ADH} 2$ or ALDH2 genotypes were not associated with hypertension.

Sensitivity analysis with adjustment for interaction of $\mathrm{ADH} 2$ / $\mathrm{ALDH} 2$ genetic polymorphism with gender, age and antihypertensive medication use also showed similar results. The interactions between $\mathrm{ADH} / \mathrm{ALDH} 2$ genetic polymorphism and these potential effect modifiers, such as gender, age and antihypertensive medication use on blood pressure levels or hypertension, were all nonsignificant ( $P$-values from 0.21 to 0.94 ).

\section{DISCUSSION}

To the best of our knowledge, this is the first study in which the association of $\mathrm{ADH} 2$ and $\mathrm{ALDH} 2$ genotypes with hypertension has been investigated in a large community-based Chinese sample. ADH2 genotype but not ALDH2 genotype was a determinant of blood pressure among alcohol drinkers; alcohol drinkers with the GG ADH2 genotype had higher blood pressure than alcohol drinkers with other genotypes, after adjusting for frequency or amount of alcohol use, suggesting that ethanol may have a key role in alcohol-induced hypertension.

Our results support studies that showed a significant interaction between $\mathrm{ADH} 2$ and alcohol drinking on blood pressure ${ }^{22}$ and no relationship between ALDH2 and blood pressure. ${ }^{22-24}$ As ALDH2 has been shown to be associated with blood pressure through affecting 
Table 3 Adjusted ORs (95\% confidence interval) of hypertension for ADH2 and ALDH2 polymorphisms in older Chinese men

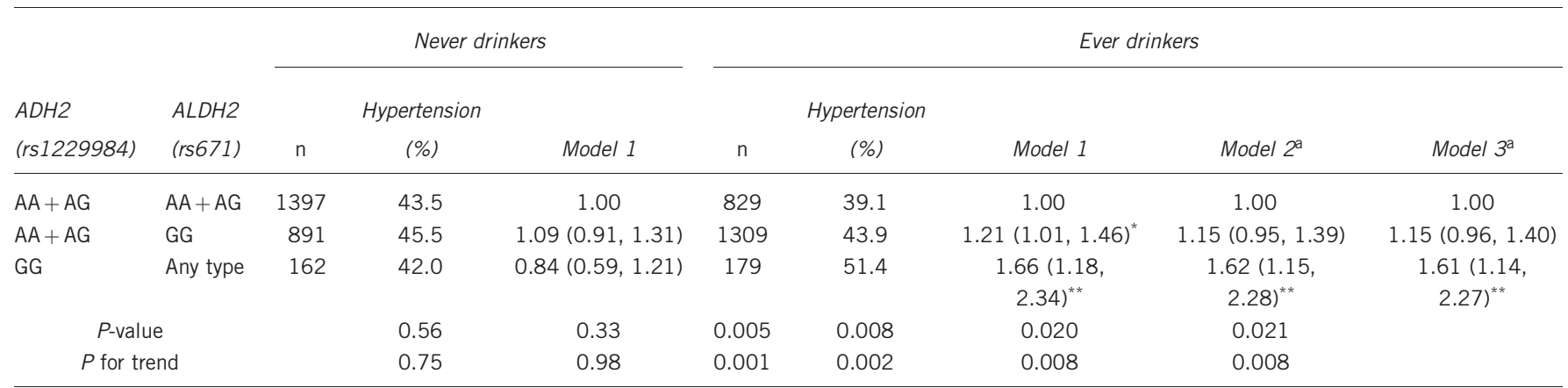

Abbreviations: ADH, alcohol dehydrogenase; ALDH, aldehyde dehydrogenase; OR, odds ratio; HDL, high-density lipoprotein.

Model 1: adjusting for age, education, occupation, smoking, physical activity, body mass index, as well as fasting total cholesterol, triglyceride, HDL cholesterol and glucose. Model 2: based on model 1 , additionally adjusting for frequency of drinking. Model 3 : based on model 1 , additionally adjusted for the amount of alcohol use ( $<$ or $\geqslant 140 \mathrm{~g}$ per week).

model 1 , additionally adjustir

a Compared with (AA/AG ADH2 + GG ALDH2), OR in GG ADH2 $=1.40(1.01,1.96), P=0.045$ in model 2 and $1.40(1.00,1.94), P=0.049$ in model 3

alcohol drinking behavior, ${ }^{5}$ the association between ALDH2 and blood pressure became statistically nonsignificant after taking into account the frequency or amount of alcohol consumption in our study. As the ALDH2 genotype influences the blood acetaldehyde level, whereas the $\mathrm{ADH} 2$ genotype influences the blood ethanol level after alcohol drinking as in Figure 1, our results suggest that the hypertensive effect of alcohol drinking is due to ethanol rather than acetaldehyde. This finding is consistent with some but not all previous studies. In an earlier study on 898 middle-aged male Japanese, no association was found between blood pressure and ADH2 or ALDH2 genotypes after adjusting for age, body mass index and volume of alcohol consumption. ${ }^{6}$ However, as the sample size was small, the null association in this study should be interpreted with caution. Another study of 335 Japanese showed that the relationship between alcohol and blood pressure was significantly stronger in those with the GG $\mathrm{ADH} 2$ genotype than those with the AA or AG genotype. ${ }^{16}$ Moreover, the authors also indicated that those with the ADH2 GG genotype were likely to develop hypertension because of excessive alcohol use. However, in our study, we have also controlled for frequency or amount of alcohol use in the model and found that older male drinkers with the GG ADH2 genotype had a higher blood pressure than alcohol drinkers with other genotypes. To remove the confounding effect of $\mathrm{ADH} 2$ genotype, we analyzed the association between ALDH2 genotypes and blood pressure in men with the AA/ AG ADH2 genotype and found no associations (table not shown), which was in accordance with previous studies ${ }^{6,16}$ showing that $\mathrm{ADH} 2$ genotype, but not ALDH2 genotype, modifies the association of alcohol with blood pressure. However, in a study of excessive male drinkers ( $>300 \mathrm{~g}$ of ethanol per week), the prevalence of the ADH2 AA genotype was significantly greater in those with higher SBP, ${ }^{22}$ suggesting that the ADH2 AA genotype but not GG genotype weakens the relationship between alcohol and blood pressure. However, participants in this study were hospital workers, included only a few heavy drinkers and had a mean age of 46 years, and the frequency or amount of alcohol use was not adjusted in the model. A confounding effect from excessive alcohol use could not be ruled out.

In the present study, we found that the ADH2 GG genotype was associated with hypertension in alcohol drinkers after adjustment for frequency of alcohol use. Hence, the relationship between ADH2 and hypertension could not be fully explained by different levels of alcohol consumption. Ethanol accumulation might be considered a key pathway. The possible mechanisms by which ethanol increases blood pressure include: (a) a direct effect on the central nervous system, by interfering with central inhibitory pathways, which control the vasomotor center or increasing sympathetic activity, ${ }^{25}$ (b) activation of the rennin-angiotensin system by ethanol, ${ }^{26}$ (c) aortic endothelial oxidative injury and downregulation of the nitric oxide generating system, ${ }^{27}$ and (d) magnesium deficiency. ${ }^{28}$ However, the actual effect of ethanol on blood pressure is unclear. Further experimental studies are warranted to examine the mechanism underlying the relationship between blood ethanol concentration and blood pressure, taking into account different $\mathrm{ADH} 2$ genotypes.

Several limitations of this study should be noted. First of all, as subjects in the present study were older Chinese people from Southern China, most of them were light to moderate drinkers. A 2001 World Health Organization sponsored survey of 24992 Chinese residents across five areas of China showed an annual ethanol consumption of 4.51 (s.d. =10.9) among adults aged 15 years and older, which was low compared with Western countries: by comparison, annual alcohol use was about 8.61 per person in 2001 in Europe. ${ }^{29}$ Moreover, people living in Southern China have lower levels of alcohol use than those in the North. ${ }^{2}$ Given the limited number of subjects in the present study who were heavy alcohol users, whether the effect of heavy drinking on hypertension varies with $\mathrm{ADH} 2$ genotype could not be assessed. Secondly, levels of alcohol use among Chinese women were too low, so we could not examine the associations among women. Thirdly, this is not a fully populationrepresentative study, and participants included were homogenous Southern Chinese from one city. Earlier studies have shown different distributions of $\mathrm{ADH} 2$ genotypes in different ethnic populations, such as Chinese, Japanese, Brazilians and African Americans. ${ }^{8,30,31}$ Further studies are needed to examine whether $\mathrm{ADH} 2$ genotypes modify the effect of alcohol on blood pressure in other ethnic groups. The strengths of the present study include a large sample size (which enabled a detailed analysis of gene-environment interactions by assessing the alcohol-blood pressure relationship in participants who were or were not alcohol drinkers), the focus on a communitybased sample with light to moderate level of alcohol use, and the adjustment of many potential confounding factors. Moreover, the current study, for the first time systematically considered ethanol metabolism in human body, including both acetaldehyde and acetic acid, and clearly showed that current drinkers with an inactive $\mathrm{ADH} 2$ genotype had a significantly increased risk of hypertension. The current study employed genetic polymorphism of an alcohol metabolism enzyme as an instrumental variable to obtain an unbiased 
estimation of the effect of alcohol on blood pressure, which was unlikely to be confounded by other risk factors, such as smoking or sodium intake, as in other observational studies. This study provided the suggestive causal inference that the hypertensive effect of alcohol drinking might be due to ethanol accumulation.

In conclusion, we found that the $\mathrm{ADH} 2$ genotype but not the ALDH2 genotype was associated with blood pressure and hypertension in current drinkers. However, in never drinkers, no association between $\mathrm{ADH} 2$ or ALDH2 genotypes and blood pressure was found, indicating a gene-environment interaction effect on alcohol-induced hypertension. Among current alcohol drinkers, individuals with a GG ADH2 genotype had a higher risk of hypertension than those with the AA or AG ADH2 genotype, suggesting a key role for ethanol in alcohol-induced hypertension. Further studies in a larger sample, including woman drinkers and younger drinkers, are warranted to confirm our results.

\section{CONFLICT OF INTEREST}

The authors declare no conflict of interest

\section{ACKNOWLEDGEMENTS}

This work was supported by the Guangzhou Public Health Bureau and the Guangzhou Science and Technology Bureau, Guangzhou, China; the University of Hong Kong Foundation for Educational Development and Research, Hong Kong; The University of Birmingham, UK; and the Guangdong Natural Science Foundation of China, Guangdong, China (Grant number: 9451062001003477).

Authors Contribution: WS Zhang and L Xu contributed to study design, data collection, data analysis and manuscript writing, CM Schooling, CQ Jiang, KK Cheng and B Liu contributed to study design and data collection, TH Lam contributed to study design, manuscript revision and approval of final submission.

1 Ma YX, Zhang B, Wang HJ, Du WW, Su C, Zhai FY. [Status and trend of alcoho consumption among adults in nine provinces (antonomous region) of China from 1993 to 2006]. Zhonghua Yu Fang Yi Xue Za Zhi 2011; 45: 323-329.

2 Cochrane J, Chen $\mathrm{H}$, Conigrave KM, Hao W. Alcohol use in China. Alcohol Alcohol. 2003; 38: 537-542.

3 Hao W, Chen H, Su Z. China: alcohol today. Addiction 2005; 100: 737-741.

4 WHO Global Status Report on Alcohol. WHO, Geneva, 1999: 9-13.

5 Chen L, Davey Smith G, Harbord RM, Lewis SJ. Alcohol intake and blood pressure: a systematic review implementing a Mendelian randomization approach. PLoS Med 2008; 5: e52.

6 Yamada Y, Sun F, Tsuritani I, Honda R. Genetic differences in ethanol metabolizing enzymes and blood pressure in Japanese alcohol consumers. J Hum Hypertens 2002 16: 479-486

7 Peng GS, Yin JH, Wang MF, Lee JT, Hsu YD, Yin SJ. Alcohol sensitivity in Taiwanese men with different alcohol and aldehyde dehydrogenase genotypes. J Formos Med Assoc 2002; 101: 769-774.

8 Bosron WF, Li TK. Genetic polymorphism of human liver alcohol and aldehyde dehydrogenases, and their relationship to alcohol metabolism and alcoholism. Hepatology 1986; 6: 502-510.

9 Hui P, Nakayama T, Morita A, Sato N, Hishiki M, Saito K, Yoshikawa Y, Tamura M, Sato I, Takahashi T, Soma M, Izumi Y, Ozawa Y, Cheng Z. Common single nucleotide polymorphisms in Japanese patients with essential hypertension: aldehyde dehydrogenase 2 gene as a risk factor independent of alcohol consumption. Hypertens Res 2007; 30: 585-592.

10 Yokoyama A, Muramatsu T, Omori T, Yokoyama T, Matsushita S, Higuchi S, Maruyama $\mathrm{K}$, Ishii $\mathrm{H}$. Alcohol and aldehyde dehydrogenase gene polymorphisms and oropharyngolaryngeal, esophageal and stomach cancers in Japanese alcoholics. Carcinogenesis 2001; 22: 433-439.

11 Yokoyama A, Muramatsu T, Omori T, Matsushita S, Yoshimizu H, Higuchi S, Yokoyama $\mathrm{T}$, Maruyama K, Ishii $\mathrm{H}$. Alcohol and aldehyde dehydrogenase gene polymorphisms influence susceptibility to esophageal cancer in Japanese alcoholics. Alcohol Clin Exp Res 1999; 23: 1705-1710.

12 Hsu LC, Tani K, Fujiyoshi T, Kurachi K, Yoshida A. Cloning of cDNAs for human aldehyde dehydrogenases 1 and 2. Proc Natl Acad Sci USA 1985; 82 3771-3775.

13 Yoshida A, Ikawa M, Hsu LC, Tani K. Molecular abnormality and cDNA cloning of human aldehyde dehydrogenases. Alcohol 1985; 2: 103-106.

14 Harada S, Agarwal DP, Goedde HW. Aldehyde dehydrogenase deficiency as cause of facial flushing reaction to alcohol in Japanese. Lancet 1981; 2: 982.

15 Yin SJ, Bosron WF, Magnes LJ, Li TK. Human liver alcohol dehydrogenase: purification and kinetic characterization of the beta 2 beta 2 , beta 2 beta 1 , alpha beta 2 , and beta 2 gamma 1 'Oriental' isoenzymes. Biochemistry 1984; 23: 5847-5853.

16 Saito K, Yokoyama T, Yoshiike N, Date C, Yamamoto A, Muramatsu M, Tanaka H. Do the ethanol metabolizing enzymes modify the relationship between alcohol consumption and blood pressure? J Hypertens 2003; 21: 1097-1105.

17 Jiang C, Thomas GN, Lam TH, Schooling CM, Zhang W, Lao X, Adab P, Liu B, Leung GM, Cheng KK. Cohort profile: The Guangzhou Biobank Cohort Study, a Guangzhou-Hong Kong-Birmingham collaboration. Int J Epidemiol 2006; 35: 844-852.

18 Thomas GN, Young RP, Tomlinson B, Anderson PJ, Woo KS, Sanderson JE, Critchley JAJH. A sibling-pair analysis of fasting lipids and anthropometric measurements and their relationship to hypertension. Clin Exp Hypertens 1999; 21: 1161-1176.

19 Liu B, Jiang CQ, Thomas GN, Lao XQ, Lin JM, Yue XJ, Zhang WS, Zhu T, Lin DQ. [Polymorphisms of vascular diseases-related genes in Guangzhou Biobank Cohort Study]. Zhonghua yu fang yi xue za zhi [Chinese journal of preventive medicine] 2010; 44: 65-69.

20 Deng HB, Macfarlane DJ, Thomas GN, Lao XQ, Jiang CQ, Cheng KK, Lam TH. Reliability and validity of the IPAQ-Chinese: the Guangzhou Biobank Cohort study. Med Sci Sport Exerc 2008; 40: 303-307.

21 Xin X, He J, Frontini MG, Ogden LG, Motsamai OI, Whelton PK. Effects of alcoho reduction on blood pressure: a meta-analysis of randomized controlled trials. Hypertension 2001; 38: 1112-1117.

22 Hashimoto Y, Nakayama T, Futamura A, Omura M, Nakarai H, Nakahara K. Relationship between genetic polymorphisms of alcohol-metabolizing enzymes and changes in risk factors for coronary heart disease associated with alcohol consumption. Clin Chem 2002; 48: 1043-1048.

23 Okayama A, Ueshima H, Yamakawa M, Kita Y. Low-Km aldehyde dehydrogenase deficiency does not influence the elevation of blood pressure by alcohol. J Hum Hypertens 1994; 8: 205-208.

24 Tsuritani I, Ikai E, Date T, Suzuki Y, Ishizaki M, Yamada Y. Polymorphism in ALDH2genotype in Japanese men and the alcohol-blood pressure relationship. Am J Hypertens 1995; 8: 1053-1059.

25 Drummond W, Shrager H. Ethanol induced dose-dependent vasoconstriction in unanesthetized lambs. Exp Lung Res 1985; 9: 341-349.

26 Fitts DA, Hoon RG. Ethanol-induced changes in plasma proteins, angiotensin II, and salt appetite in rats. Behav Neurosci 1993; 107: 339-345.

27 Husain K. Vascular endothelial oxidative stress in alcohol-induced hypertension. Cell Mol Biol (Noisy-le-grand) 2007; 53: 70-77.

28 Hsieh ST, Sano H, Saito K, Kubota Y, Yokoyama M. Magnesium supplementation prevents the development of alcohol-induced hypertension. Hypertension 1992; 19: $175-182$.

29 Hao W, Su Z, Liu B, Zhang K, Yang H, Chen S, Biao M, Cui C. Drinking and drinking patterns and health status in the general population of five areas of China. Alcohol Alcohol 2004; 39: 43-52.

30 Goedde HW, Agarwal DP, Fritze G, Meier-Tackmann D, Singh S, Beckmann G, Bhatia K, Chen LZ, Fang B, Lisker $\mathrm{R}$ et al. Distribution of ADH2 and ALDH2 genotypes in different populations. Hum Genet 1992; 88: 344-346.

31 Iron A, Groppi A, Fleury B, Begueret J, Cassaigne A, Couzigou P. Polymorphism of class I alcohol dehydrogenase in French, Vietnamese and Niger populations: genotyping by PCR amplification and RFLP analysis on dried blood spots. Ann Genet 1992; 35: $152-156$.

(c) (i) (5) $\odot$ This work is licensed under a Creative Commons Attribution-NonCommercial-NoDerivs 3.0 Unported License. To view a copy of this license, visit http://creativecommons. org/licenses/by-nc-nd/3.0/ 